\title{
Genome-wide Sequencing in Rheumatic Diseases
}

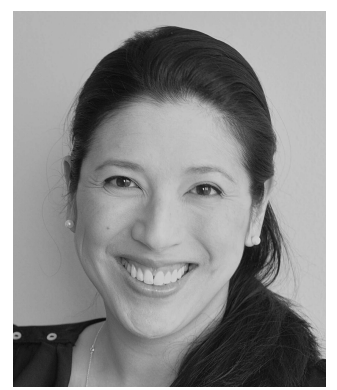

As next-generation whole exome sequencing (WES) and whole genome sequencing (WGS) become increasingly available and affordable, their application has led to the growing identification of monogenic forms of rheumatic diseases, traditionally recognized as complex diseases. This is demonstrated in Batu, et al's paper, "Whole Exome Sequencing in Early-onset Systemic Lupus Erythematosus," appearing in this issue of The Journal ${ }^{1}$.

The authors completed WES on 7 Turkish patients with systemic lupus erythematosus (SLE), with disease onset at 5 years of age or younger, from multiplex families (proband had an affected sibling) or who were offspring of consanguineous parents. They found 5 patients who were homozygous for variants predicted to alter the early complement cascade proteins. The sixth patient was homozygous for a 2-base pair deletion in DNASE1L3, a variant previously associated with young-onset SLE and hypocomplementemic urticarial vasculitis ${ }^{2,3,4}$. The seventh patient was homozygous for a number of variants, with an HDAC7 variant deemed a potentially causal variant.

The Batu, et al paper ${ }^{1}$ highlights how the identification of causal genetic variants leading to monogenic lupus not only provides insights into the probable pathogenic variants responsible for disease and rare forms of monogenic lupus, but that these variants also implicate pathogenic mechanisms in SLE more broadly. One such example is DNASE1L3, an enzyme responsible for clearance of genetic material from apoptotic cellular debris. It is predicted that the reduced DNASE1L3 activity results in impaired clearance of self-DNA, becoming an antigenic stimulus leading to the production of autoantibodies including anti-DNA antibodies and SLE. A DNASE1L3 knockout (-/-) mouse rapidly developed anti-dsDNA and antichromatin antibodies and an SLE phenotype ${ }^{5}$. Interestingly, compared to healthy controls, patients with SLE (who presumably do not carry rare variants in DNASE1L3) are observed to have both decreased DNASE1L3 protein levels $(1000 \mathrm{pg} / \mathrm{ml}$ vs $2250 \mathrm{pg} / \mathrm{ml}$, $p=0.003)$ and enzyme activity (60\% vs $90 \%$ activity, $\mathrm{p}<0.001)$ in the serum. In patients with SLE, lower DNASE1L3 levels were associated with higher indices of disease activity $(685.1 \pm 624.1 \mathrm{pg} / \mathrm{ml}$ with active SLE vs $1761.0 \pm 1441.4 \mathrm{pg} / \mathrm{ml}$ with inactive SLE, $\mathrm{p}=0.04)$ and nephritis $(686.5 \pm 557.5 \mathrm{pg} / \mathrm{ml}$ with nephritis vs $1893.4 \pm$ $1504.0 \mathrm{pg} / \mathrm{ml}$ without nephritis, $\mathrm{p}=0.02)^{6}$.

In the arena of clinical genetics, obtaining a genetic diagnosis often does not lead to improved therapeutic options but rather is important for excluding treatable disorders and to inform family planning and anticipatory guidance to family members, as well as to provide an end to the diagnostic odyssey ${ }^{7}$. In contrast, a genetic diagnosis in immunologic and rheumatic diseases may have critical implications for therapy, and the potential for improved patient outcomes. This is exemplified in the identification of genetic variants leading to increased interleukin (IL)-1 $\beta$ secretion in cryopyrin-associated periodic syndromes or cryopyrinopathies. The genetic link contributed to the identification of appropriate candidate patients for IL-1 blockade, which dramatically improved patients' lives by controlling disease activity and preventing harmful organ damage 8,9 .

Outlined below are some of the more common options for genetic testing, a brief description of when each may be indicated, and the advantages and limitations of each test.

\section{Targeted gene panel}

Gene panels provide a relatively inexpensive and rapid first test of known genes associated with disease, and usually contain 10-200 genes. In accordance with the American College of Medical Genetic and Genomics guidelines, only genes with known associations with disease should be included in gene panels ${ }^{10}$. Panels can provide high read depth in known disease-associated genes, and the limited number of variants eases analysis and interpretability in the clinical context. Gene panels are typically limited to coding sequence variants (i.e., exons) and are sometimes complemented by multiplex ligation-dependent probe amplification

See Exome sequencing in SLE, page 1671 
for detection of copy-number variants $(\mathrm{CNV})$ and insertion/deletions of typically $<50$ base pairs. Another limitation is that gene panels still have regions of low coverage such as guanine- and cytosine-rich regions, and will not identify larger insertions and deletions. Therefore, negative gene panels often necessitate subsequent WES or WGS to identify variants.

\section{WES}

The exome is the protein-coding portion of the genome: it comprises about $2 \%$ of the human genome. The exome is estimated to harbor $85 \%$ of disease-causing variants ${ }^{11}$. Hence, WES provides an affordable, unbiased examination of the part of the genome most likely to yield results. Increasingly, laboratories are completing WES but interpreting only genes with known associations with disease, as part of panel testing ${ }^{10}$. By examining the entire exome, it is possible to prioritize known genes and easily expand examination to other genes for potential discovery of novel gene-disease associations. In a study of 250 unselected, consecutive patients who had WES, likely causal variants were identified in 62 patients, achieving a $25 \%$ diagnostic yield ${ }^{12}$. Another study of 145 pediatric patients concluded that $23 \%$ of patients diagnosed by WES would have been missed by commercial gene panels, because the implicated genes were omitted from the panels ${ }^{13}$. Similar to panel testing, WES may be complemented by chromosomal microarray for CNV detection, another form of genetic variation. One of the limitations of WES is the potential to miss large genetic deletions/duplications that may be responsible for disease. Also, the causal genetic variation may not localize to the protein coding regions of the genome. Both of these limitations are overcome with examination of the whole genome.

\section{WGS}

WGS enables unbiased examination of virtually all types of genetic variation, including noncoding and structural variants. Similar to WES, WGS allows the option to begin with a prioritized examination of genes of interest, which can be expanded easily to search across the entire genome.

In 2015, the Canadian College of Medical Geneticists published a position statement recommending clinical genome-wide sequencing (WES and/or WGS) as an appropriate approach in the diagnosis of patients suspected of a monogenic disease ${ }^{14}$. The listed factors that increase the likelihood of monogenic disease include family history (i.e., a recognizable pattern of inheritance, consanguinity), severe phenotype, and negative prior genetic tests such as chromosomal microarray or gene panels.

It has been demonstrated that WGS achieves a 3-fold increased diagnostic yield for pediatric diseases when compared to standard of care chromosome microarray, and subsequent gene-specific sequencing ${ }^{15,16}$, particularly for neurologic disorders and/or congenital anomalies.
The clinical utility of genome-wide sequencing including whole exome and whole genome sequencing is only now being elucidated because early studies conducting genome-wide sequencing were primarily in the research setting. Only recently have studies examined the clinical and cost-effective application of genome-wide sequencing. For rheumatic diseases, one of our first challenges is in identifying those individuals most suitable for WES and WGS. There is certainly a great opportunity to gain further insights into the biologic pathways of disease, the identification of novel therapeutic targets, and ultimately improved care and outcomes for patients and families.

LINDA T. HIRAKI, MD, FRCPC, ScD, Hospital for Sick Children, Rheumatology, 555 University Ave.,

Toronto, Ontario M5G 1X5, Canada.

Address correspondence to Dr. L.T. Hiraki.

E-mail: linda.hiraki@sickkids.ca

\section{REFERENCES}

1. Batu ED, Koşukcu C, Taşkıran E, Sahin S, Akman S, Sözeri B, et al. Whole exome sequencing in early-onset systemic lupus erythematosus. J Rheumatol 2018;45:1671-9.

2. Al-Mayouf SM, Sunker A, Abdwani R, Abrawi SA, Almurshedi F, Alhashmi N, et al. Loss-of-function variant in DNASE1L3 causes a familial form of systemic lupus erythematosus. Nat Genet 2011;43:1186-8.

3. Ozcakar ZB, Foster J 2nd, Diaz-Horta O, Kasapcopur O, Fan YS, Yalcinkaya F, et al. DNASE1L3 mutations in hypocomplementemic urticarial vasculitis syndrome. Arthritis Rheum 2013;65:2183-9.

4. Carbonella A, Mancano G, Gremese E, Alkuraya FS, Patel N, Gurrieri F, et al. An autosomal recessive DNASE1L3-related autoimmune disease with unusual clinical presentation mimicking systemic lupus erythematosus. Lupus 2017;26:768-72.

5. Sisirak V, Sally B, D’Agati V, Martinez-Ortiz W, Ozcakar ZB, David J, et al. Digestion of chromatin in apoptotic cell microparticles prevents autoimmunity. Cell 2016;166:88-101.

6. Zhao Q, Yang C, Wang J, Li Y, Yang P. Serum level of DNase113 in patients with dermatomyositis/polymyositis, systemic lupus erythematosus and rheumatoid arthritis, and its association with disease activity. Clin Exp Med 2017;17:459-65.

7. Hayeems RZ, Boycott KM. Genome-wide sequencing technologies A primer for paediatricians. Paediatr Child Health 2018;23:191-7.

8. Gattorno M, Martini A. Treatment of autoinflammatory syndromes. Curr Opin Pediatr 2010;22:771-8.

9. Lane T, Loeffler JM, Rowczenio DM, Gilbertson JA, Bybee A, Russell TL, et al. AA amyloidosis complicating the hereditary periodic fever syndromes. Arthritis Rheum 2013;65:1116-21.

10. Rehm HL, Bale SJ, Bayrak-Toydemir P, Berg JS, Brown KK, Deignan JL, et al. ACMG clinical laboratory standards for $\mathrm{n}$ ext-generation sequencing. Genet Med 2013;15:733-47.

11. Majewski J, Schwartzentruber J, Lalonde E, Montpetit A, Jabado N. What can exome sequencing do for you? J Med Genet 2011; 48:580-9.

12. Yang Y, Muzny DM, Reid JG, Bainbridge MN, Willis A, Ward PA, et al. Clinical whole-exome sequencing for the diagnosis of mendelian disorders. N Engl J Med 2013;369:1502-11.

13. Dillon OJ, Lunke S, Stark Z, Yeung A, Thorne N, Melbourne Genomics Health Alliance, et al. Exome sequencing has higher diagnostic yield compared to simulated disease-specific panels in

Personal non-commercial use only. The Journal of Rheumatology Copyright (c) 2018. All rights reserved. 
children with suspected monogenic disorders. Eur J Hum Genet 2018;26:644-51.

14. Boycott K, Hartley T, Adam S, Bernier F, Chong K, Fernandez BA, et al. The clinical application of genome-wide sequencing for monogenic diseases in Canada: position statement of the Canadian College of Medical Geneticists. J Med Genet 2015;52:431-7.

15. Lionel AC, Costain G, Monfared N, Walker S, Reuter MS, Hosseini $\mathrm{SM}$, et al. Improved diagnostic yield compared with targeted gene sequencing panels suggests a role for whole-genome sequencing as a first-tier genetic test. Genet Med 2018;20:435-43.

16. Stavropoulos DJ, Merico D, Jobling R, Bowdin S, Monfared N, Thiruvahindrapuram $\mathrm{B}$, et al. Whole genome sequencing expands diagnostic utility and improves clinical management in pediatric medicine. NPJ Genom Med 2016;1:15012.

J Rheumatol 2018;45:1614-16; doi:10.3899/jrheum.180951 